\title{
SYMMETRIC ORTHOGONAL POLYNOMIALS AND THE ASSOCIATED ORTHOGONAL L-POLYNOMIALS
}

\author{
A. SRI RANGA
}

(Communicated by Hal L. Smith)

\begin{abstract}
We show how symmetric orthogonal polynomials can be linked to polynomials associated with certain orthogonal L-polynomials. We provide some examples to illustrate the results obtained. Finally as an application, we derive information regarding the orthogonal polynomials associated with the weight function $\left(1+k x^{2}\right)\left(1-x^{2}\right)^{-1 / 2}, k>0$.
\end{abstract}

\section{INTRODUCTION}

Let $\mathbf{Y}(n)$ be the space of all real monic polynomials $P_{n}(x)$ of degree $n$ that satisfy the symmetric property $P_{n}(x)=(-1)^{n} P_{n}(-x)$. Let $\mathrm{Z}(n, \beta)$ be the space of all real monic polynomials $Q_{n}(t)$ of degree $n$ that satisfy the rather different symmetric property $Q_{n}(t)=t^{n} Q_{n}\left(\beta^{2} / t\right) /(-\beta)^{n}$.

It is well known (see $[1,13])$ that if $w(x)$ is a weight function on $(-d, d)$, where $0<d \leq \infty$, such that $w(x)=w(-x)$, then the associated monic orthogonal polynomials $B_{n}(w ; x), n \geq 0$, are such that $B_{n}(w ; x) \in \mathbf{Y}(n)$. Furthermore, they satisfy

$$
B_{n+1}(w ; x)=x B_{n}(w ; x)-\alpha_{n+1}(w) B_{n-1}(w ; x), \quad n \geq 1,
$$

where

$$
\alpha_{n+1}(w)=\frac{\int_{-d}^{d} x^{n} B_{n}(w ; x) w(x) d x}{\int_{-d}^{d} x^{n-1} B_{n-1}(w ; x) w(x) d x}>0, \quad n \geq 1 .
$$

Now, some lesser known results are the following. Let $\nu(t)$ be a strong weight function defined on $\left(\beta^{2} / b, b\right)$, where $0<\beta<b \leq \infty$, such that

$$
\sqrt{t} \nu(t)=\sqrt{\beta^{2} / t} \nu\left(\beta^{2} / t\right) .
$$

Then the monic polynomials $\widetilde{B}_{n}(\nu ; t), n \geq 0$, defined uniquely by

$$
\int_{\beta^{2} / b}^{b} t^{-n+s} \widetilde{B}_{n}(\nu ; t) \nu(t) d t=0, \quad 0 \leq s \leq n-1,
$$

Received by the editors March 29, 1994.

1991 Mathematics Subject Classification. Primary 33C45, 42C05.

Key words and phrases. Orthogonal polynomials, L-polynomials, recurrence relations.

This work was supported by the Brazilian research foundations $\mathrm{CNP}_{\mathrm{q}}$ and FAPESP.

(c) 1995 American Mathematical Society 
are such that $\widetilde{B}_{n}(\nu ; t) \in \mathbf{Z}(n, \beta)$ and satisfy

$$
\widetilde{B}_{n+1}(\nu ; t)=(t-\beta) \widetilde{B}_{n}(\nu ; t)-\tilde{\alpha}_{n+1}(\nu) t \widetilde{B}_{n-1}(\nu ; t), \quad n \geq 1,
$$

where

$$
\tilde{\alpha}_{n+1}(\nu)=\frac{\int_{\beta^{2} / b}^{b} \widetilde{B}_{n}(\nu ; t) \nu(t) d t}{\int_{\beta^{2} / b}^{b} \widetilde{B}_{n-1}(\nu ; t) \nu(t) d t}>0, \quad n \geq 1 .
$$

For proofs, and other information on these results, see [8, 12]. In [9, 10, 11, 12] some results can be found regarding the polynomials defined by (1.3) for weight functions satisfying a property different from (1.2). For some studies of polynomials given by a variation of $(1.3)$, see $[5,6,7]$.

It is easily verified from (1.3) that the Laurent polynomials or L-polynomials given by

$$
R_{2 m}(t)=t^{-m} \widetilde{B}_{2 m}(\nu ; t), \quad R_{2 m+1}(t)=t^{-m-1} \widetilde{B}_{2 m+1}(\nu ; t), \quad m \geq 0,
$$

form a sequence of orthogonal L-polynomials on $\left(\beta^{2} / b, b\right)$ in relation to the strong weight function $\nu(t)$. For information on orthogonal L-polynomials and polynomials similar to $\widetilde{B}_{n}(\nu ; t)$, but where $\nu(t)$ is any strong weight function, we refer to $[2,3,4]$.

In this article we show the relation between the polynomials $B_{n}(w ; x)$ and $\widetilde{B}_{n}(\nu ; t)$, when the associated weight functions are linked in a certain way. We also provide some examples to illustrate the results obtained. Finally as an application, we derive information regarding the orthogonal polynomials $B_{n}(W ; x), n \geq 0$, where $d=1$ and $W(x)=\left(1+k x^{2}\right)\left(1-x^{2}\right)^{-1 / 2}$, for $k>0$.

\section{Preliminary Results}

Throughout the rest of this article we assume $\alpha>0$ and $\beta>0$.

Theorem 2.1. Let the sequence of polynomials $\left\{P_{n}(x)\right\}$ be such that $P_{n}(x) \in$ $\mathbf{Y}(n)$. Then

$$
P_{n}(x)=B_{n}(w ; x), \quad n \geq 0,
$$

if and only if

(2.1)

$$
\int_{-d}^{d}\left\{\sqrt{\alpha x^{2}+\beta}+\sqrt{\alpha} x\right\}^{-(n-1)+2 s} \frac{P_{n}(x)}{\sqrt{\alpha x^{2}+\beta}} w(x) d x=0, \quad 0 \leq s \leq n-1 .
$$

Proof. Since $\left\{\sqrt{\alpha x^{2}+\beta}+\sqrt{\alpha} x\right\}^{-1}=\left\{\sqrt{\alpha x^{2}+\beta}-\sqrt{\alpha} x\right\} / \beta,(2.1)$ is equivalent to

$$
\int_{-d}^{d}\left\{\sqrt{\alpha x^{2}+\beta} \pm \sqrt{\alpha} x\right\}^{2 l} \frac{P_{2 m+1}(x)}{\sqrt{\alpha x^{2}+\beta}} w(x) d x=0
$$

and

$$
\int_{-d}^{d}\left\{\sqrt{\alpha x^{2}+\beta} \pm \sqrt{\alpha} x\right\}^{2 l+1} \frac{P_{2 m+2}(x)}{\sqrt{\alpha x^{2}+\beta}} w(x) d x=0
$$

for $l=0,1, \ldots, m$ and $m \geq 0$. 
Using the symmetric property of $P_{n}(x)$, this can also be given in the equivalent form

$$
\begin{aligned}
& \int_{-d}^{d}\left[\sum_{r=0}^{l-1}\left(\begin{array}{c}
2 l \\
2 r+1
\end{array}\right)\left\{\alpha x^{2}+\beta\right\}^{l-r-1}\{ \pm \sqrt{\alpha} x\}^{2 r+1}\right] P_{2 m+1}(x) w(x) d x=0, \\
& \int_{-d}^{d}\left[\sum_{r=0}^{l}\left(\begin{array}{c}
2 l+1 \\
2 r
\end{array}\right)\left\{\alpha x^{2}+\beta\right\}^{l-r}\{ \pm \sqrt{\alpha} x\}^{2 r}\right] P_{2 m+2}(x) w(x) d x=0,
\end{aligned}
$$

for $l=0,1, \ldots, m$ and $m \geq 0$.

Now, if $P_{n}(x)=B_{n}(w ; x)$ for $n \geq 0$, then (2.2) must hold as $B_{n}(w ; x)$ is orthogonal to all polynomials of degree $\leq n$.

Conversely, if (2.2) holds, then, for example, $P_{2 m+1}(x)$ is orthogonal to the odd polynomials

$$
\sum_{r=0}^{l-1}\left(\begin{array}{c}
2 l \\
2 r+1
\end{array}\right)\left\{\alpha x^{2}+\beta\right\}^{l-r-1}\{\sqrt{\alpha} x\}^{2 r+1},
$$

of "precise" degree $2 l-1$, for $l=0,1, \ldots, m$. Since these polynomials must form a basis for all odd polynomials of degree $\leq 2 m-1, P_{2 m+1}(x)$ must be orthogonal to all odd polynomials of degree $\leq 2 m-1$. Hence, as $P_{2 m+1}(x)$ is monic and is also odd, we obtain $P_{2 m+1}(x)=B_{2 m+1}(w ; x)$. In a similar way we also show that $P_{2 m}(x)=B_{2 m}(w ; x)$. This completes the proof of the theorem.

We now consider the transformation

$$
t(x)=\left\{\sqrt{\alpha x^{2}+\beta}+\sqrt{\alpha} x\right\}^{2}, \quad x \in(-\infty, \infty) .
$$

The transformation $t(x)$ represents a one-to-one correspondence between $(-\infty, \infty)$ and $(0, \infty)$. The inverse of $t(x)$ is

$$
x(t)=\frac{1}{2 \sqrt{\alpha}}(\sqrt{t}-\beta / \sqrt{t}), \quad t \in(0, \infty) .
$$

If $x=d$ corresponds to $t=b$, that is, if $\sqrt{b}=\sqrt{\alpha d^{2}+\beta}+\sqrt{\alpha} d$, then it is easily seen that $x=-d$ corresponds to $t=\beta^{2} / b$.

Theorem 2.2. With $x(t)$ given by (2.3), let $Q(t)=(2 \sqrt{\alpha t})^{n} P(x(t))$. Then

$$
P(x) \in \mathbf{Y}(n) \text { iff } Q(t) \in \mathbf{Z}(n, \beta) .
$$

Proof. Since $x\left(\beta^{2} / t\right)=-x(t)$, it follows that

$$
\frac{t^{n} Q\left(\beta^{2} / t\right)}{(-\beta)^{n}}=(-1)^{n}(2 \sqrt{\alpha t})^{n} P(-x(t)) .
$$

Hence, $P(x)=(-1)^{n} P(-x)$ iff $Q(t)=t^{n} Q\left(\beta^{2} / t\right) /(-\beta)^{n}$. Thus, all we need to show is that if $P(x)$ is a monic polynomial of degree $n$, then so is $Q(t)$ and vice versa. This can be established using the following observations.

If $Q(t) \in \mathbf{Z}(n, \beta)$ and if $Q(t)=\sum_{r=0}^{n} q_{r} t^{r}$, where $q_{n}=1$, then $q_{n-r}=$ $(-\beta)^{2 r-n} q_{r}$. Hence, for $r=[(n+1) / 2], \ldots, n$

$$
\{2 \sqrt{\alpha t(x)}\}^{-n}\left[q_{r}\{t(x)\}^{n}+q_{n-r}\{t(x)\}^{n-r}\right]
$$


is a polynomial of the form

$$
(4 \alpha)^{r-n} q_{r} x^{2 r-n}+\text { lower degrees. }
$$

On the other hand, if $P(x) \in \mathbf{Y}(n)$, then for $n=2 m$ we can write $P(x)=$ $\sum_{r=0}^{m} p_{2 r} x^{2 r}$, where $p_{2 m}=1$. Hence, $(2 \sqrt{\alpha t})^{2 m} p_{2 r}\{x(t)\}^{2 r}$ is the polynomial

$$
(4 \alpha)^{m-r} p_{2 r} t^{m-r}(t-\beta)^{2 r},
$$

for $r=0,1, \ldots, m$. Similarly for $n=2 m+1$, we can write $P(x)=$ $\sum_{r=0}^{m} p_{2 r+1} x^{2 r+1}$, where $p_{2 m+1}=1$. Hence, $(2 \sqrt{\alpha t})^{2 m+1} p_{2 r+1}\{x(t)\}^{2 r+1}$ is the polynomial

$$
(4 \alpha)^{m-r} p_{2 r+1} t^{m-r}(t-\beta)^{2 r}, \quad r=0,1, \ldots, m .
$$

\section{THE MAIN RESULtS}

We first consider a result regarding the weight functions.

Theorem 3.1. Let $b$ and $d$ be such that $\sqrt{b}=\sqrt{\alpha d^{2}+\beta}+\sqrt{\alpha} d$ and let

$$
V(t)=A t^{-1 / 2} W(x(t)) .
$$

Then $W(x)$ is a weight function on $(-d, d)$ such that $W(x)=W(-x)$ if and only if $V(t)$ is a strong weight function on $\left(\beta^{2} / b, b\right)$ such that $\sqrt{t} V(t)=$ $\sqrt{\beta^{2} / t} V\left(\beta^{2} / t\right)$. Here, $A$ is any positive number.

Proof. It is easily verified that if $W(x)$ is positive and satisfies $W(x)=W(-x)$ in $(-d, d)$, then $V(t)$ is positive and satisfies $\sqrt{t} V(t)=\sqrt{\beta^{2} / t} V\left(\beta^{2} / t\right)$ in $\left(\beta^{2} / b, b\right)$ and vice versa. Therefore we need only to prove that when the moments (including the negative ones for $V(t)$ ) exist and are finite for one of the functions, the same is also true for the other function. We have

$$
\int_{\beta^{2} / b}^{b} t^{r} V(t) d t=A 2 \sqrt{\alpha} \int_{-d}^{d}\left\{\sqrt{\alpha x^{2}+\beta}+\sqrt{\alpha} x\right\}^{2 r+1} \frac{W(x)}{\sqrt{\alpha x^{2}+\beta}} d x .
$$

Since $W(x)=W(-x)$, this can be written as

$$
\int_{\beta^{2} / b}^{b} t^{r} V(t) d t=\int_{-d}^{d} S_{r}(x) W(x) d x,
$$

where $S_{r}(x)$ is an even polynomial of exact degree $2 r$ for $r \geq 0$ and is an even polynomial of exact degree $|2 r|-2$ for $r \leq-1$. As the monomials $x^{2 r}$ and the polynomials $S_{r}(x)$ can be expressed as linear combinations of each other, the proof of the theorem follows.

Theorem 3.2. Let $W(x)$ and $V(t)$ be a pair of weight functions given by Theorem 3.1. Then for $n \geq 0$

$$
\widetilde{B}_{n}(V ; t)=(2 \sqrt{\alpha t})^{n} B_{n}(W ; x(t)) .
$$

Proof. From Theorems 2.1 and 3.1 it follows that

$$
\int_{\beta^{2} / b}^{b} t^{-n+s}(2 \sqrt{\alpha t})^{n} B_{n}(W ; x(t)) V(t) d t=0, \quad 0 \leq s \leq n-1 .
$$

Hence from (1.3) and Theorem 2.2, the result of the theorem is immediate. 
As a consequence of (3.1) we obtain from (1.1) and (1.4) that

$$
\tilde{\alpha}_{n+1}(V)=4 \alpha \alpha_{n+1}(W), \quad n \geq 1 .
$$

Furthermore, if $G(x, u)$ is a generating function for the polynomials $B_{n}(W ; x)$, then

$$
F(t, u)=G(x(t), 2 \sqrt{\alpha t} u)
$$

is a generating function for $\widetilde{B}_{n}(V ; t)$.

\section{ILLUSTRATIVE EXAMPLES}

Example 1. The Tchebyshev case. If $W(x)=1 / \sqrt{1-x^{2}}$ in $(-1,1)$, then from Theorem 3.1

$$
V(t)=\frac{1}{\sqrt{b-t} \sqrt{t-a}} \text { in }(a, b),
$$

where $\sqrt{b}=\sqrt{\alpha+\beta}+\sqrt{\alpha}$ and $\sqrt{a}=\sqrt{\alpha+\beta}-\sqrt{\alpha}$.

The associated polynomials $\widetilde{B}_{n}(V ; t), n \geq 1$, and their interpolatory quadrature rules have already been studied in [8]. From [8], we can also confirm that (3.2) holds.

Now from (3.3) we get

$$
F(t, u)=\frac{1-(t-\beta) u}{1-2(t-\beta) u+4 \alpha t u^{2}},
$$

as a generating function for $\widetilde{B}_{n}(V ; t)$.

Example 2. The Gegenbauer case. For $\lambda>0$, if $W(x)=\left(1-x^{2}\right)^{\lambda-1 / 2}$ in $(-1,1)$, then

$$
V(t)=t^{-\lambda}(b-t)^{\lambda-1 / 2}(t-a)^{\lambda-1 / 2} \text { in }(a, b),
$$

where $\sqrt{b}=\sqrt{\alpha+\beta}+\sqrt{\alpha}$ and $\sqrt{a}=\sqrt{\alpha+\beta}-\sqrt{\alpha}$. From the recurrence relation for the monic Gegenbauer polynomials it then follows that

$$
\tilde{\alpha}_{n+1}(V)=\frac{n(n+2 \lambda-1)}{(n+\lambda)(n+\lambda-1)} \alpha, \quad n \geq 1 .
$$

For $\lambda=1 / 2$, that is, for the Legendre case, this result has already been given in [8].

From (3.3), we obtain as a generating function for $\widetilde{B}_{n}(V ; t)$,

$$
F(t, u)=\left\{1-2(t-\beta) u+4 \alpha t u^{2}\right\}^{-\lambda} .
$$

Example 3. The Hermite case. If $W(x)=\exp \left(-x^{2}\right)$ in $(-\infty, \infty)$, then

$$
V(t)=t^{-1 / 2} \exp \left(-\frac{\left(t+\beta^{2} / t\right)}{4 \alpha}\right) \quad \text { in }(0, \infty) .
$$

From the recurrence relation for the monic Hermite polynomials we find

$$
\tilde{\alpha}_{n+1}(V)=2 \alpha n, \quad n \geq 1 .
$$

This result for $\alpha=1 / 2$ has also been given in [8].

Using (3.3) as a generating function for $\widetilde{B}_{n}(V ; t)$ we have

$$
F(t, u)=\exp \left(2(t-\beta) u-4 \alpha t u^{2}\right) .
$$




\section{AN APPLication}

Let $W(x)=\left(1+k x^{2}\right)\left(1-x^{2}\right)^{-1 / 2}$ in $(-1,1)$, where $k>0$. Choosing $\alpha$ and $\beta$ such that $k=\alpha / \beta$, we obtain

$$
V(t)=\frac{t^{-1}(t+\beta)^{2}}{\sqrt{b-t} \sqrt{t-a}}=\frac{t(1+\beta / t)^{2}}{\sqrt{b-t} \sqrt{t-a}} \text { in }(a, b),
$$

where $\sqrt{b}=\sqrt{\alpha+\beta}+\sqrt{\alpha}$ and $\sqrt{a}=\sqrt{\alpha+\beta}-\sqrt{\alpha}$. Equivalently, we can also write $\beta=\sqrt{a b}$ and $\sqrt{\alpha}=(\sqrt{b}-\sqrt{a}) / 2$.

It has been shown in [12] that the polynomials $\widetilde{B}_{n}(V ; t)$ satisfy the recurrence relation (1.4) with

$$
\tilde{\alpha}_{n+1}(V)=\frac{K_{n-1} K_{n+2}}{K_{n} K_{n+1}} \alpha, \quad n \geq 1,
$$

with $K_{n}=(1+l)^{n}+(1-l)^{n}, n \geq 0$, and $l=\sqrt{1+\alpha / \beta}$. Hence from (3.2) we obtain the following result.

Theorem 5.1. The monic orthogonal polynomials $B_{n}(W ; x), n \geq 0$, associated with the weight function

$$
W(x)=\frac{1+k x^{2}}{\sqrt{1-x^{2}}} \text { in }(-1,1),
$$

where $k>0$, satisfy

$$
B_{n+1}(W ; x)=x B_{n}(W ; x)-\frac{1}{4} \frac{K_{n-1} K_{n+2}}{K_{n} K_{n+1}} B_{n-1}(W ; x), \quad n \geq 1,
$$

with $B_{0}(W ; x)=1, B_{1}(W ; x)=x$. Here $K_{n}=(1+l)^{n}+(1-l)^{n}, n \geq 0$, and $l=\sqrt{1+k}$.

Since $\int_{-1}^{1} W(x) d x=(2+k) \pi / 2$, we obtain from (5.1) the orthogonality property

$$
\int_{-1}^{1} B_{m}(W ; x) B_{n}(W ; x) \frac{1+k x^{2}}{\sqrt{1-x^{2}}} d x=\delta_{m n} 2^{-2 n} \frac{K_{n+2}}{K_{n}} \pi / 2 .
$$

Finally, if we let $P_{n}(W ; x)=2^{n-1} K_{n} B_{n}(W ; x)$ for $n \geq 0$, then

$$
\int_{-1}^{1} P_{m}(W ; x) P_{n}(W ; x) \frac{1+k x^{2}}{\sqrt{1-x^{2}}} d x=\delta_{m n} K_{n} K_{n+2} \pi / 8
$$

and

$$
K_{n} P_{n+1}(W ; x)=2 K_{n+1} x P_{n}(W ; x)-K_{n+2} P_{n-1}(W ; x), \quad n \geq 1,
$$

with $P_{0}(W ; x)=1$ and $P_{1}(W ; x)=2 x$.

\section{REFERENCES}

1. T. S. Chihara, An introduction to orthogonal polynomials, Math. Appl., Gordon and Breach, New York, 1978.

2. W. B. Jones, O. Njảstad, and W. J. Thron, Two-point Padé expansions for a family of analytic functions, J. Comput. Appl. Math. 9 (1983), 105-123.

3. W. B. Jones, W. J. Thron, and H. Waadeland, A strong Stieltjes moment problem, Trans. Amer. Math. Soc. 261 (1980), 503-528. 
4. O. Njảstad and W. J. Thron, The theory of sequences of L-polynomials, Padé Approximants and Continued Fractions (H. Waadeland and H. Wallin, eds.), Det Kongelige Norsk Videnskabers Selskab, vol. 1, Universitetsforlaget, Trondeihm, 1983, pp. 54-91.

5. A. Sri Ranga, On a recurrence formula associated with strong distributions, SIAM J. Math. Anal. 21 (1990), 1335-1348.

6. A. Sri Ranga and J. H. McCabe, On the extensions of some classical distributions, Proc. Edinburgh Math. Soc. 34 (1991), 61-67.

7. A. Sri Ranga, The strong c-symmetric distribution, J. Austral. Math. Soc. Ser. A 53 (1992), 61-65.

8. _ Another quadrature rule of highest algebraic degree of precision, Numer. Math. 68 (1994), 283-294.

9. A. Sri Ranga and E. X. L. de Andrade, $A$ weight function that appears in the limit and certain associated polynomals (submitted).

10. A. Sri Ranga, E. X. L. de Andrade, and J. H. McCabe, Some consequences of symmetry in strong distributions, J. Math. Anal. Appl. (to appear).

11. A. Sri Ranga and C. F. Bracciali, A continued fraction associated with a special Stieltjes function, Comm. Anal. Theory Continued Fractions 3 (1994), 60-64.

12. A. Sri Ranga and J. H. McCabe, On pairwise related strong Stieltjes distributions (submitted).

13. G. Szegö, Orthogonal polynomials, 4th ed., Amer. Math. Soc. Colloq. Publ., vol. 23, Amer. Math. Soc., Providence, RI, 1975.

Departamento de CiênCias de Computação e Estatística, IBILCE, Universidade Estadual Paulista (UNESP), 15054-000 São José do Rio Preto, SP, Brazil

E-mail address: ranga@nimitz.ibilce.unesp.br 